\title{
Detecting bubbles in exotic nuclei
}

\author{
E. Khan, M. Grasso, J. Margueron, N. Van Giai ${ }^{1}$ \\ ${ }^{1}$ Institut de Physique Nucléaire, Université Paris-Sud, IN2P3-CNRS, F-91406 Orsay Cedex, France
}

\begin{abstract}
The occurrence of a bubble, due to an inversion of $\mathrm{s}_{1 / 2}$ state with the state usually located above, is investigated. Proton bubbles in neutron-rich Argon isotopes are optimal candidates. Pairing effects which can play against the bubble formation are evaluated. They cannot prevent bubble formation in very neutron-rich argon isotopes such as ${ }^{68} \mathrm{Ar}$. This pleads for a measurement of the charge density of neutron-rich argon isotopes in the forthcoming years, with the advent of electron scattering experiments in next generation exotic beam facilities such as FAIR or RIBF.
\end{abstract}

PACS numbers: 21.10.Ft, 21.10Re, 21.60.Jz, 25.30.Bf, 25.70.De

\section{INTRODUCTION}

The occurrence of bubble nuclei has been studied by Wilson in 1946 [1], in order to describe low-lying excitations as oscillations of nuclear bubbles. The invoked reason for the bubble existence was an overestimated "saturation of the nuclear forces". Bethe and Siemens have also investigated this possibility in the sixties [2]. The stability of the bubble was studied using the liquid drop model and this analysis showed that bubbles were not leading to the minimum energy configuration, compared to prolate shape with uniform density. During the seventies and eighties, bubble nuclei were also investigated, still with liquid drop based models [3] or Thomas-Fermi approaches [4]. More recently bubbles were predicted in hyperheavy nuclei [5] with $\mathrm{Z} \geq 120$. In this case, selfconsistent Hartree-Fock-Bogoliubov (HFB) calculations have shown that the ground state corresponds to a bubble configuration. This effect is due to an interplay between the Coulomb interaction and the nucleon-nucleon interaction in those very heavy systems. The optimum spatial position of the bubble has also been investigated [6]. However the existence of such heavy nuclei is speculative, and bubbles have not been detected yet. It should be noted that bubble effects may be a general feature in nuclei: they have also been predicted in superheavy nuclei around $\mathrm{Z}=120$ [7] using mean-field calculations.

A qualitatively different interpretation for the bubble existence is invoking a specific microscopic quantum mechanical effect: the s-states being the only wave functions with non zero value at $r=0$, the depopulation of this level leads to a depletion of the density in the center of the nucleus. Such an interior-peaked shape of the $3 \mathrm{~s}$ wave function has been measured using electron scattering on ${ }^{206} \mathrm{~Pb}$ and ${ }^{205} \mathrm{Tl}$ [8]. However, the depletion in the interior of these nuclei remains small since in heavy nuclei the $2 \mathrm{~s}_{1 / 2}$ state is already filled and contributes to the density at $\mathrm{r}=0$. In an early Hartree-Fock + BCS calculation a strong depletion in the interior of ${ }^{36} \mathrm{Ar}$ and ${ }^{200} \mathrm{Hg}$ nuclei was predicted [9], which the authors defined as a bubble occurrence. However, their effective nucleon-nucleon interaction was not considered as sound, and it was shown that using more elaborated interactions prevent bubble formation in ${ }^{36} \mathrm{Ar}[10]$. It should be noted that the key point for the bubble formation in the s-d region is the inversion of the proton $2 \mathrm{~s}_{1 / 2}$ and $1 \mathrm{~d}_{3 / 2}$ states with respect to the standard shell model.

The emergence of experimental studies on exotic nuclei gathered numerous signals of shell structure modification these last decades (see e.g. [11, 12, 13]). It is therefore legitimate to suspect that in more neutron-rich nuclei, the s and d state crossing may occur. Such an inversion was recently predicted in the ${ }^{46} \mathrm{Ar}$ nucleus using relativistic mean field (RMF) calculations. A strong depletion in the center of the proton density was obtained [14]. However pairing effects could preclude the bubble effect due to the occupancy of $2 \mathrm{~s}_{1 / 2}$ state. Such a study has been extended to $\mathrm{N}=28$ isotones [15]. The mechanism of this inversion in neutron-rich nuclei has been analyzed in Ref. [16], and it is due to the conjunction of several factors: the spinorbit potential modification, the tensor force, as well as the lowering of the proton potential and the extension of the proton density due to the presence of a neutron skin. Predictions for more neutron-rich nuclei show an increase of the gap between the inverted s and d states, strongly supporting the formation of proton bubbles in ${ }^{60-68} \mathrm{Ar}$.

In this paper we analyze the general features of bubble occurrence in exotic nuclei, on an illustrative case. The bubble occurrence is a direct consequence of the $\mathrm{s}_{1 / 2}$ depopulation, which could be obtained either by lowering the proton number, or by a level inversion involving an s state. This work is devoted to the latter case. The possible candidates are reviewed in Section II, showing that proton bubbles in very neutron-rich Argon isotopes are the most likely. Several experimental methods to detect such a bubble are then investigated : charge density measurements (Section III) and direct reactions (Section IV).

\section{CANDIDATE NUCLEI}

\section{A. Possible candidates}

Bubble candidates are nuclei where the state is depopulated. In some cases this occurs due to an inversion between the s state and the one usually located above. Therefore, either proton or neutron states inversion be- 


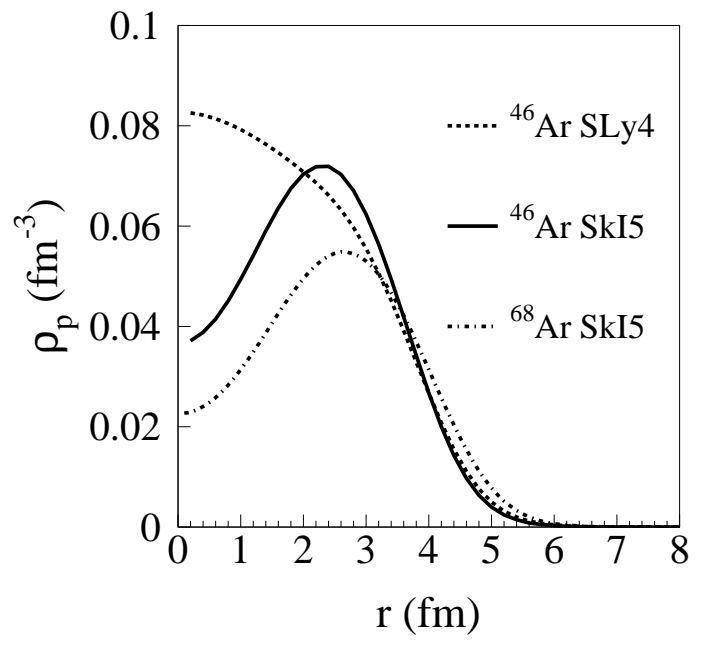

FIG. 1: Proton densities of ${ }^{46} \mathrm{Ar}$ calculated with the SkI5 interaction (full lines) and SLy4 [20] (dashed lines) in the HF approach. The ${ }^{68} \mathrm{Ar}$ proton density calculated with the SkI5 interaction is also shown (dashed-dotted lines)

tween $\left(2 \mathrm{~s}_{1 / 2}, 1 \mathrm{~d}_{3 / 2}\right)$ states or $\left(3 \mathrm{~s}_{1 / 2}, 1 \mathrm{~h}_{11 / 2}\right)$ states are possible candidates. In the proton bubble case, these inversions correspond to $\mathrm{Ar}$ and $\mathrm{Hg}$ isotopes. In the neutron bubble case, they correspond to the $\mathrm{N}=18$ and $\mathrm{N}=80$ isotones. A first experimental signal for an inversion may be given by the $\mathrm{J}^{\pi}$ of the ground state of the odd nuclei having one additional nucleon compared to the bubble nuclei ( $\mathrm{N}$ or $\mathrm{Z}$ equals to 19 or 81 ). A $\mathrm{J}^{\pi}=1 / 2^{+}$together with a spectroscopic factor close to one is an indication for an $\mathrm{s}_{1 / 2}$ state located above the usual $1 \mathrm{~d}_{3 / 2}$ or $1 \mathrm{~h}_{11 / 2}$ state. In the case of the Potassium isotopes, such values have been measured in ${ }^{47} \mathrm{~K}$ [17], showing that the s-d states are almost degenerated. An $\left(2 \mathrm{~s}_{1 / 2}, 1 \mathrm{~d}_{3 / 2}\right)$ inversion is even predicted by RMF calculations [14]. In Ref. [16], the study along the Calcium isotopic chain shows that the s-d states inversion is strengthened for very neutronrich nuclei $(\mathrm{A} \geq 58)$. Hence the study of very neutron-rich Argon isotopes such as ${ }^{68} \mathrm{Ar}$ using the HF method provides an illustrative study of the bubble manifestation. Fig. 1 shows the proton densities of ${ }^{68} \mathrm{Ar}$, calculated with spherical HF model using the SkI5 [18] force. The numerical details are the same as in Ref. [16]. A strong depletion in obtained in the center of the nucleus, with this SkI5 parametrization. It should be noted that the large s-d state inversion for very neutron-rich nuclei in the ${ }^{68} \mathrm{Ar}$ region is also obtained with several other interactions [16] as well as shell-model calculations [19].

In the case of the $\mathrm{N}=18$ isotones, the measured $\mathrm{J}^{\pi}$ values of the ground states of the $\mathrm{N}=19$ isotones are $\mathrm{J}^{\pi}=3 / 2^{+}$[21], showing no clear signal for an inversion.
On the contrary all the measured $\mathrm{J}^{\pi}$ of the $\mathrm{Z}=81$ isotopes are $1 / 2^{+}$in the ground state, showing a systematic inversion leading to a possible proton bubble in the $\mathrm{Hg}$ isotopes. However, as stated above, the depletion in the center of the proton density of the $\mathrm{Hg}$ isotopes is less pronounced than for the Argon isotopes, since in heavy nuclei the $2 \mathrm{~s}_{1 / 2}$ state is already filled and contributes to the density at $\mathrm{r}=0$. For the $\mathrm{N}=81$ isotones, $\mathrm{J}^{\pi}$ are measured for only 3 nuclei and $\mathrm{J}^{\pi}=1 / 2^{+}$is found in ${ }^{145} \mathrm{Gd}$ and ${ }^{147} \mathrm{Dy}$ [21]. In this case also, the bubble effect in heavy nuclei is expected to be less important than in intermediate mass nuclei. To summarize, the optimal manifestation of a bubble could be for protons in neutron-rich Argon isotopes. We will therefore focus on these nuclei in the following.

It should be noted that deformation can play against a bubble occurrence: the degeneracy removal attenuates the bubble effect generated by the inoccupancy of the $2 \mathrm{~s}_{1 / 2}$ state. In the case of ${ }^{46} \mathrm{Ar}$, several $\mathrm{N}=28$ studies used deformed mean-field models 22, 23, 24], and also the Generator Coordinate Method (GCM) based on Gogny force [25]. In the HFB calculations, ${ }^{46} \mathrm{Ar}$ is predicted as a soft nucleus, either spherical or with a small deformation parameter in the ground state. More neutron-rich Argon isotopes are also predicted spherical [24]. In the GCM approach, the ground state of ${ }^{46} \mathrm{Ar}$ is predicted as a possible shape coexistence state. In the following we will consider the spherical case for both ${ }^{46} \mathrm{Ar}$ and ${ }^{68} \mathrm{Ar}$ nuclei in order to study the bubble hypothesis in this framework.

\section{B. Pairing effects}

Pairing effects may hinder the bubble formation since scattered pairs could populate the $\mathrm{s}_{1 / 2}$ state, decreasing the depletion in the center of the nucleus. The occupancy factor of the s-state due to pairing effects may be modified. The study of the proton pairing effect in Argon isotopes is a delicate task : it is occurring in a two-hole state from a doubly magic nucleus. It is known that the HFB approximation is not well designed to such a situation, leading to an overestimation of the pairing effects. For instance, we have performed HFB calculations on ${ }^{46,68} \mathrm{Ar}$ using the SkI5 interaction and a delta density-dependent pairing interaction, of the form :

$$
V_{\text {pair }}=V_{0}\left[1-\eta\left(\frac{\rho(r)}{\rho_{0}}\right)^{\alpha}\right] \delta\left(\mathbf{r}_{1}-\mathbf{r}_{\mathbf{2}}\right)
$$

with $\eta=1, \alpha=0.5$ and $\rho_{0}=0.16 \mathrm{fm}^{3}$. The magnitude $\mathrm{V}_{0}=-330 \mathrm{MeV} \cdot \mathrm{fm}^{3}$ of the pairing interaction is obtained by reproducing the two-proton separation energy of ${ }^{46} \mathrm{Ar}$. Fig. 2 displays the proton density of ${ }^{68} \mathrm{Ar}$, showing a reduction of the bubble effect, but still a depletion is predicted in the center of the nucleus.

In ${ }^{46} \mathrm{Ar}$ the pairing interaction plays an even more dramatic role: the s state occupation is predicted to be $54 \%$. 


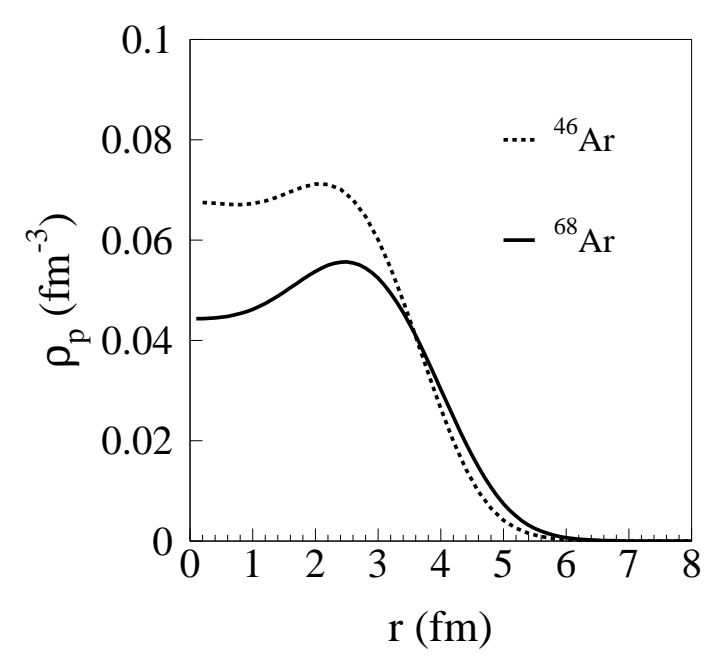

FIG. 2: Proton densities of ${ }^{46} \mathrm{Ar}$ (dashed lines) and ${ }^{68} \mathrm{Ar}$ calculated with the SkI5 interaction in the HFB approach.

The corresponding proton densities, with and without pairing effects, are displayed on Fig. 1 and 2, respectively. As expected the $1 \mathrm{~d}_{3 / 2}$ state is depopulated, due to the pairing effect. Shell-model calculations also predict around $50 \%$ occupation probability for the $2 \mathrm{~s}_{1 / 2}$ state [19]. However, it should be noted that the $2 \mathrm{~s}_{1 / 2}$ and $1 \mathrm{~d}_{3 / 2}$ occupation probabilities have not been measured yet, leaving a small possibility for a bubble occurrence in ${ }^{46} \mathrm{Ar}$.

In Ref. [14] the splitting of the neutron p-states in ${ }^{46} \mathrm{Ar}$ is weakened by the proton density depletion in the center of the nucleus, which modifies the spin-orbit term in the interior: the proton depletion is related to the neutron splitting of the p orbits measured in Ref. [19, 26]. The weakening of the neutron $p$ states splitting with the occupancy of the $2 \mathrm{~s} 1 / 2$ orbital has been recently studied in [15] from $\mathrm{Si}$ to $\mathrm{Ca} \mathrm{N}=28$ isotones. A proton depletion is predicted for nuclei having a low $2 \mathrm{~s}_{1 / 2}$ occupation probability.

The presence of a proton bubble in ${ }^{46} \mathrm{Ar}$ is subject to significant uncertainties, due to the dependence on the pairing and the Skyrme interactions. Hence, experimental indications of a bubble in this nucleus are of relevant importance. The probability of a proton bubble manifestation increases when going from ${ }^{46} \mathrm{Ar}$ to much more neutron-rich nuclei such as ${ }^{60-68}$ Ar. As mentioned above, several interactions used in Ref. [16] predict an inversion between the $2 \mathrm{~s}_{1 / 2}$ and $1 \mathrm{~d}_{3 / 2}$ proton states, with a large gap around ${ }^{70} \mathrm{Ca}$, showing that the proton bubble prediction for very neutron-rich Argon isotopes is a general feature.
In the following we choose ${ }^{46} \mathrm{Ar}$ as an illustrative example. We will therefore consider the bubble hypothesis through the HF predictions in ${ }^{46} \mathrm{Ar}$ using the SkI5 interaction (Fig. 1). The no-bubble hypothesis can be obtained either by SLy4-HF (Fig. 1) or by SkI5-HFB (Fig. 2) calculations. We have checked that the two latter cases give very similar results: in Ref. [16] HF calculations using SLy4 do not predict any inversion of the s and d states, implying no bubble. Hence we will describe the no-bubble hypothesis using the HF calculations with the SLy 4 interaction. The case of ${ }^{46} \mathrm{Ar}$ is chosen only for illustrative purposes since experimental data are available on this nucleus. All conclusions below remain valid in the case of ${ }^{68} \mathrm{Ar}$.

\section{PROBING THE CHARGE DENSITY}

Although there are strong indications that the $2 \mathrm{~s}_{1 / 2}$ is half filled in ${ }^{46} \mathrm{Ar}$, we choose this nucleus to illustrate experimental signals associated with the bubble occurrence, as explained above. It should be noted that similar conclusions could be drawn on ${ }^{68} \mathrm{Ar}$ or other bubble nuclei with respect to the present calculations. ${ }^{46} \mathrm{Ar}$ is an unstable nucleus which can be produced in present exotic beam facilities with a typical intensity of $10^{3}$ pps. Several experimental studies have already been performed and we will analyze in the following these data with respect to a bubble manifestation. The most direct observable related to a proton bubble is the charge density.

\section{A. The charge radius}

Charge radii of Ar isotopes have been precisely measured from ${ }^{32} \mathrm{Ar}$ to ${ }^{46} \mathrm{Ar}[27,28]$, using laser spectroscopy of fast beams at the ISOLDE isotope separator. The obtained value for ${ }^{46} \mathrm{Ar}$ is $\mathrm{R}_{c}=3.44 \pm 0.01 \mathrm{fm}$. However, the calculated ${ }^{46} \mathrm{Ar}$ r.m.s. charge radii with the HF or HFB models are mainly sensitive to the surface part of the proton density, and are not adequate quantities to detect a bubble in the center of the nucleus: a typical variation of $0.02 \mathrm{fm}$ is found between calculations assuming the bubble and the no-bubble hypothesis. This difference is not enough to lead to a clear signature of a bubble occurrence. It is therefore necessary to look for complementary observables which could be more sensitive to a bubble manifestation.

\section{B. Electron scattering}

The ideal experiment to probe the charge density would be electron scattering. Presently electrons scattering on unstable nuclei like ${ }^{46} \mathrm{Ar}$ is not possible, but it is expected in next generation facilities such as RIBF in Riken [29] of FAIR in GSI [30]. In this case accelerated electrons would scatter on a radioactive beam of 


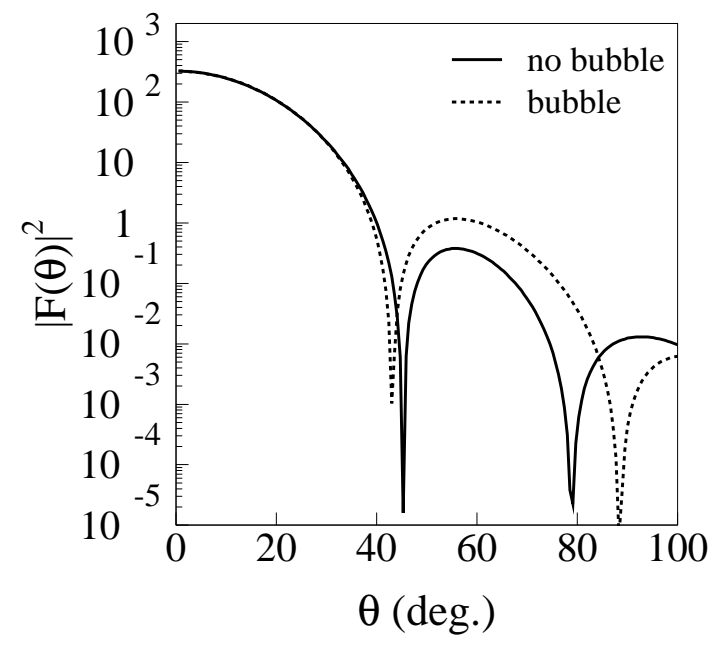

FIG. 3: Angular dependence of the form factor obtained for $300 \mathrm{MeV}$ electron scattering on ${ }^{46} \mathrm{Ar}$, using either the HF-SkI5 density (dashed line) or the HF-SLy4 density (solid line)

${ }^{46} \mathrm{Ar}$ kept in a storage ring. Such an experiment may be feasible in the next decade. It is therefore worthwhile to check how accurately the electron scattering experiment could probe the proton bubble presence. We have performed calculation for $300 \mathrm{MeV}$ electron scattering ${ }^{46} \mathrm{Ar}$. Fig. 3 shows the angular dependence of the form factor defined by :

$$
F(q)=\int \rho_{c}(r) e^{i \vec{q} \cdot \vec{r}} d \vec{r}
$$

where $\rho_{c}$ is the charge density calculated with HF model using SLy4 or SkI5 interactions, and q is the transferred momentum, related to the incident momentum $\mathrm{p}$ and the scattering angle $\theta$ :

$$
q=2 p \sin \frac{\theta}{2}
$$

Both the no-bubble and the bubble cases exhibit a diffraction pattern. However, above 45 deg. the angular distribution is located at higher magnitude in the case of a bubble than without a bubble. Around $80 \mathrm{deg}$. an angular shift between the two distributions is noticed, the minima of the bubble distribution being $10 \mathrm{deg}$. larger than the normal one. At 90 deg. the two angular distributions are in opposite phases, showing that this angle is the optimum one to disentangle between the two hypothesis. At larger angle the angular shift increases but the magnitude becomes too small for a clear measurement. In summary, an optimal experiment to search for a bubble in very neutron-rich Argon isotopes would be elastic electron scattering at $300 \mathrm{MeV}$, focused on scattered electrons between 40 degrees and 100 degrees.

\section{DIRECT REACTIONS AND COLLECTIVE MODES}

As stated above, such an experiment with electron scattering on exotic beams is not feasible yet. It is therefore interesting to analyze what hints direct reactions could provide with respect to a bubble manifestation. It is well known that direct reactions probe the surface of the nucleus, so it is not possible to directly detect a bubble located in the center of the nucleus. However, in the Argon isotopes, the bubble is a straightforward consequence of the inversion between the $1 \mathrm{~d}_{3 / 2}$ and the $2 \mathrm{~s}_{1 / 2}$ levels, provided that the $2 \mathrm{~s}_{1 / 2}$ remains depopulated. Direct reactions are the most accurate tool to study these features. For instance transfer reactions such as $\left(\mathrm{d},{ }^{3} \mathrm{He}\right)$ would allow to measure the spectroscopic factors associated to the $2 \mathrm{~s}_{1 / 2}$ level. Collective modes such as giant resonances and low-lying states may also provide useful information about a bubble manifestation. We shall again illustrate the bubble occurrence on ${ }^{46} \mathrm{Ar}$, which could more probably occur on more neutron-rich isotopes.

\section{A. Giant resonances}

Giant resonances are collective modes involving all the nucleons. To disentangle the effect of the bubble from the one due to the interaction, namely due to the modification of the single particle spectrum, HF and HFB calculations have also been performed with both the SLy4 and the SkI5 interactions in the ${ }^{48} \mathrm{Ca}$ case, where no bubble effect is present.

In order to perform such a study and compare with the data, HFB+QRPA calculations have been performed. All detailed presentation of the model can be found in Ref. [31]. The residual interaction is derived from the Skyrme functional, as well as the pairing residual interaction, derived from the pairing part of the functional used in the HFB calculation. We have first checked that the HFB+QRPA results using SkI5 give results similar to the HF+RPA using SLy4, that is the no-bubble hypothesis. Therefore it is sufficient to perform calculations using SLy4 without pairing effects. The Landau-Migdal approximation is used, leading to a small breaking of the self-consistency, cured by a renormalization of the residual interaction to set the center of mass spurious mode to zero energy [31]. The typical renormalization values are 0.9 .

The main effects on giant resonances of ${ }^{46} \mathrm{Ar}$ which are not present in ${ }^{48} \mathrm{Ca}$ are obtained in the low energy part of the dipole and monopole resonances (Fig 44). No strong discrepancy is observed between the bubble and the normal cases in this low energy area. In the case of 

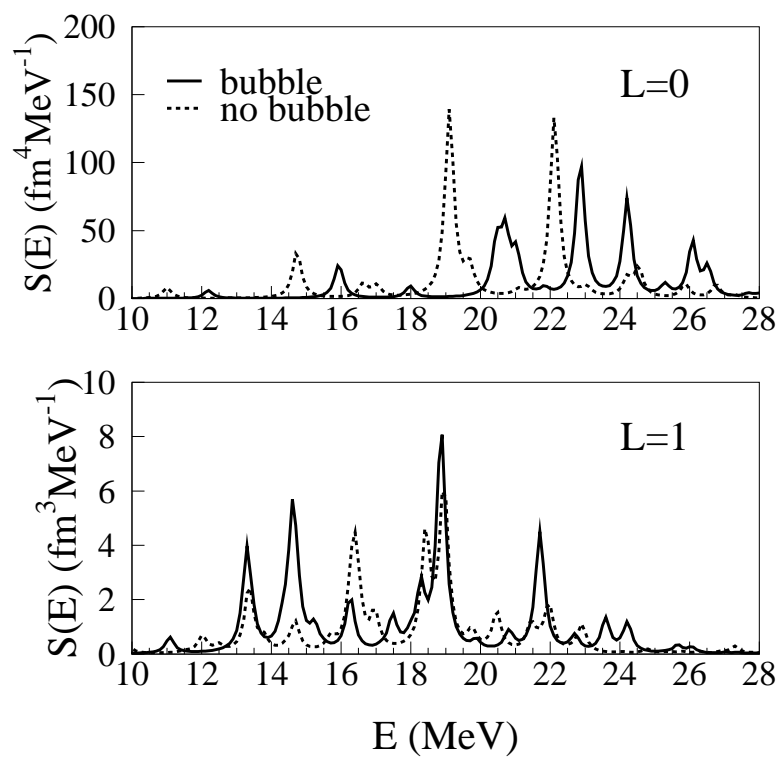

FIG. 4: Isoscalar monopole and isovector dipole strength functions obtained with the HF+RPA approach and the SkI5 interaction (solid line) or the SLy4 interaction (dashed line) in ${ }^{46} \mathrm{Ar}$

the monopole response, the lowest energy state is shifted by $1.5 \mathrm{MeV}$ at higher energy in the bubble case, from $14.5 \mathrm{MeV}$, to $16 \mathrm{MeV}$. It may be interpreted as a slight increase of the nucleus incompressibility for a soft mode in the bubble case: protons are depleted in the center of the nucleus and are redistributed in the surface, making more difficult a soft compression of the nucleus.

The isovector E1 strength exhibits an increase of the low-lying part, around $13 \mathrm{MeV}$, in the bubble case. This effect is not present in the SkI5 calculations on ${ }^{48} \mathrm{Ca}$ : the strength enhancement is more important in the ${ }^{46} \mathrm{Ar}$ case than in the ${ }^{48} \mathrm{Ca}$ case. This soft mode may be interpreted as the oscillation of neutron with respect to protons which are surface localized.

However, the above mentioned effects are hardly sizable, especially for giant resonances related effects, where the spreading width implies a large experimental width which can mask these effects.

\section{B. Low-lying states}

The low-lying states of ${ }^{46} \mathrm{Ar}$ have been recently studied using direct reactions, either by Coulomb excitation [32] or by proton scattering in inverse kinematics [33]. As stated above, they are an accurate tool to study the level inversion as well as the eventual population of the $2 \mathrm{~s}_{1 / 2}$ state due to the pairing effect.

The study of the first $2^{+}$state of ${ }^{46} \mathrm{Ar}$ by proton scattering shows a significant contribution of the neutrons to the excitation [33]. Hence this state is not the best probe for the proton shell structure. However, the study of the proton contribution through the $\mathrm{B}(\mathrm{E} 2)$ value could provide relevant information. Fig. 5 shows the isoscalar quadrupole response of ${ }^{46} \mathrm{Ar}$, calculated by $\mathrm{HF}+\mathrm{RPA}$ with the SLy4 interaction (no bubble) and the SkI5 one (bubble). There is a strong enhancement of the strength of the $2_{1}^{+}$state in the bubble case. The predicted B(E2) values strongly differs: $24 \mathrm{e}^{2} \cdot \mathrm{fm}^{4}$ in the normal case and $256 \mathrm{e}^{2} \cdot \mathrm{fm}^{4}$ in the bubble case. This last case is in very good agreement with the data 32] : $\mathrm{B}(\mathrm{E} 2)=218 \pm 31$ $\mathrm{e}^{2} \cdot \mathrm{fm}^{4}$. This enhancement is partly due to the inversion between the $1 \mathrm{~d}_{3 / 2}$ and $2 \mathrm{~s}_{1 / 2}$ states: more proton particlehole configurations contribute in the bubble case than in the normal case. However, the main effect comes from the RPA residual interaction, which is very different in the two cases for this low-lying mode. Namely, the densitydependent terms of the residual interaction could explain this change, since the density profile is different in the two cases. Moreover as stated above, HFB+QRPA calculations using the SkI5 interaction, give similar results to the normal case, showing that it is the density dependence of the residual interaction which drives the $\mathrm{B}(\mathrm{E} 2)$ enhancement. These terms are generated by the densitydependent part of the effective nucleon-nucleon interaction itself. Therefore the study of bubble nuclei could not only provide information on the spin-orbit term as stated in Ref. [14, 15], but also on the importance of the densitydependent term of the nucleon-nucleon interaction. The measurement of the $\mathrm{B}(\mathrm{E} 2)$ in bubble nuclei may open a way to explore the impact of this term in the energy density functional. This shows that the manifestation of a bubble in ${ }^{46} \mathrm{Ar}$ might be necessary to reproduce the data, namely the first $2^{+}$state.

\section{CONCLUSIONS}

We have investigated the bubble occurrence in nuclei, due to an inversion of the $\mathrm{s}_{1 / 2}$ state with the state usually located above. The neutron-rich Argon isotopes are the best candidates of the nuclear chart, since the proton $2 \mathrm{~s}_{1 / 2}$ state largely contributes to the density in the center of the nucleus, and because the inversion effect is known to increase with the neutron number. Very neutron-rich nuclei such as ${ }^{68} \mathrm{Ar}$ are optimal candidates. In less neutron-rich nuclei such as ${ }^{46} \mathrm{Ar}$, the situation is less favorable since the $\left(2 \mathrm{~s}_{1 / 2}, 1 \mathrm{~d}_{3 / 2}\right)$ inversion is reduced, implying a significant occupation probability for the $2 \mathrm{~s}_{1 / 2}$ state, leading to a weakening of the bubble effect.

${ }^{46} \mathrm{Ar}$ is chosen as an illustrative case, considering both the bubble and no-bubble hypothesis: experimental data are available on this nucleus, and the predictions are similar for more neutron-rich isotopes. The p-states neutron splitting may be related to a proton depletion, and the $\mathrm{B}(\mathrm{E} 2)$ is also very well reproduced in ${ }^{46} \mathrm{Ar}$, considering the bubble hypothesis. These indications call for a more detailed experimental investigation in this nu- 


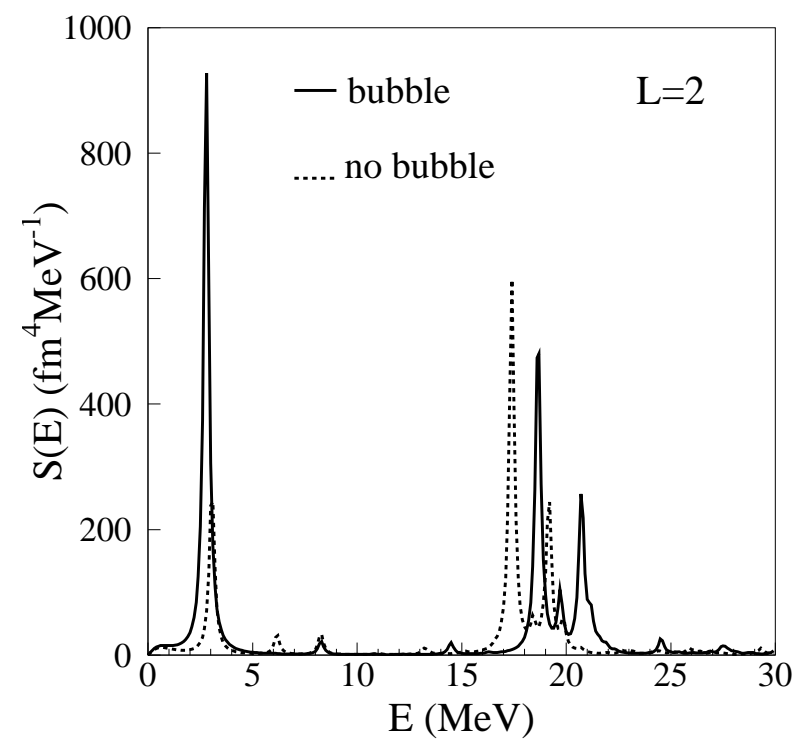

FIG. 5: Isoscalar quadrupole strength function obtained with the HF+RPA approach and the SkI5 interaction (solid line) or the SLy4 interaction (dashed lines) in ${ }^{46} \mathrm{Ar}$ cleus, as well as more neutron-rich ones, which could be undertaken by elastic electron scattering with the next generation exotic beam facilities such as FAIR or RIBF.

The bubble effect is weakened by pairing effects which can populate the $2 \mathrm{~s}_{1 / 2}$ state. However the inverted $\mathrm{s}$ and d level spacing increases for very neutron-rich Argon nuclei, and even in the case of a strong pairing, some isotopes more neutron-rich than ${ }^{46} \mathrm{Ar}$ should exhibit a bubble, as predicted in the dramatic case of ${ }^{68}$ Ar. Hence, direct reactions on ${ }^{60,62} \mathrm{Ar}$ in next generation radioactive beam facilities will also be of great interest. They should focus on low-lying states since giant resonances do not exhibit strongly different patterns in the case of a bubble, compared to the normal case.

The discovery of bubbles in nuclei is certainly an important issue for nuclear structure. This study calls for other theoretical investigations, as well as an important experimental program devoted to frontline exotic nuclei, such as neutron-rich argon isotopes.

Acknowledgments The authors thank F. Leblanc, O. Sorlin and D. Verney for fruitful discussions.
[1] H.A. Wilson, Phys. Rev. 69 (1946) 538.

[2] P. Siemens, H.A. Bethe, Phys. Rev. Lett. 18 (1967) 704.

[3] W.J. Swiatecki, Phys. Script. 28 (1983) 349.

[4] O. Bohigas, X. Campi, H. Krivine, J. Treiner, Phys. Lett. B64 (1976) 381.

[5] J. Dechargé, J.-F Berger, M. Girod, K. Dietrich, Nucl. Phys. A716 (2003) 55.

[6] Y. Yu, A. Bulgac, P. Magierski, Phys. Rev. Lett. 84 (2000) 412.

[7] M. Bender, K. Rutz, P.-G. Reinhard, J.A. Maruhn, W. Greiner, Phys. Rev. C60 (2003) 034304

[8] J.M. Cavedon et al., Phys. Rev. Lett 49 (1982) 978.

[9] X. Campi and D.W.L. Sprung, Phys. Lett. B46 (1973) 291.

[10] M. Beiner and R.J. Lombard, Phys. Lett. B47 (1973) 399.

[11] A. Ozawa, T. Kobayashi, T. Suzuki, K. Yoshida, I. Tanihata, Phys. Rev. Lett 84 (2000) 5493.

[12] E. Becheva et al., Phys. Rev. Lett 96 (2006) 012501.

[13] Z. Elekes et al., Phys. Rev. Lett 98 (2007) 102502.

[14] B.G. Todd-Rudel, J. Piekarewicz, P.D. Cottle, Phys. Rev. C69 (2004) 021301(R).

[15] J. Piekarewicz, J. Phys. G34 (1996) 467.

[16] M. Grasso, Z. Ma, E. Khan, J. Margueron, Nguyen Van Giai, Phys. Rev. C76 (2007) 044319.

[17] C.A. Ogilvie et al., Nucl. Phys. A465 (1987) 445

[18] P.G. Reinhard and H. Flocard, Nucl. Phys. A584 (1995) 467

[19] L. Gaudefroy et al., Phys. Rev. Lett. 99 (2007) 099202

[20] E. Chabanat, P. Bonche, P. Haensel, J. Meyer, R. Schaeffer, Nucl. Phys. A635 (1998) 231.
[21] R.B. Firestone, Table of isotopes 1996, Wiley Interscience Ed.

[22] T.R. Werner et al., Nucl. Phys. A597 (1996) 327

[23] G.A. Lalazissis, D. Vretenar, P. Ring, M. Stoitsov, and L. M. Robledo, Phys. Rev. C60 (1999) 014310

[24] S. Hilaire, M. Girod, http://www-phynu.cea.fr/ science_en_ligne/carte_potentiels_microscopiques/ carte_potentiel_nucleaire.htm (2006)

[25] R. Rodriguez-Guzman, J.L. Egido, L.M. Robledo, Phys. Rev. C65 (2002) 024304

[26] L. Gaudefroy et al., Phys. Rev. Lett. 97 (2006) 092501.

[27] A. Klein et al., Nucl. Phys. A607 (1996) 1

[28] K. Blaum et al., Hyperfine Interactions 162 (2005) 101

[29] T. Motobayashi, Recent Achievements and Perscpetive in Nuclear Physics, Proc. of the Vth Italy-Japan Symposium, Naples (2005) 563

[30] B. Rubio and T. Nilsson, Nucl. Phys. News 16 (2006) 5

[31] E. Khan, N. Sandulescu, M. Grasso, N. V. Giai, Phys. Rev C66 (2002) 024309.

[32] A. Gade et al., Phys. Rev. C68 (2003) 014302

[33] L.A. Rileyet al., Phys. Rev. C72 (2005) 024311 